\title{
DSMC GRID METHODOLOGIES FOR COMPUTING LOW-DENSITY, HYPERSONIC Flows About Reusable LAUnCh Vehicles
}

\author{
Richard G. Wilmoth* \\ NASA Langley Research Center \\ Hampton, VA 23681-0001 \\ Gerald J. LeBeau ${ }^{\dagger}$ \\ NASA Johnson Space Center \\ Houston, TX 77058-3696 \\ Ann B. Carlson ${ }^{\ddagger}$ \\ NASA Langley Research Center \\ Hampton, VA 23681-0001
}

\begin{abstract}
$\underline{\text { Abstract }}$
Two different grid methodologies are studied for application to DSMC simulations about reusable launch vehicles. One method uses an unstructured, tetrahedral grid while the other uses a structured, variable-resolution Cartesian grid. The relative merits of each method are discussed in terms of accuracy, computational efficiency, and overall ease of use. Both methods are applied to the computation of a low-density, hypersonic flow about a winged single-stage-to-orbit reusable launch vehicle concept at conditions corresponding to an altitude of 120 $\mathrm{km}$. Both methods are shown to give comparable results for both surface and flowfield quantities as well as for the overall aerodynamic behavior. For the conditions simulated, the flowfield about the vehicle is very rarefied but the DSMC simulations show significant departure from free-molecular predictions for the surface friction and heat transfer as well as certain aerodynamic quantities.

\section{Nomenclature}

$\begin{array}{ll}\text { CAD } & \text { Computer Aided Design } \\ \text { C.G. } & \text { Center of Gravity } \\ \mathrm{C}_{\mathrm{m}} & \text { pitching moment about C.G. }\end{array}$

\footnotetext{
* Senior Research Engineer, Aerothermodynamics Branch, Gas Dynamics Division, Senior Member, AIAA

$\dagger$ Research Engineer, Aeroscience Branch, Aeroscience and Flight Mechanics Division.

† Senior Research Engineer, Radiation Sciences Branch, Atmospheric Sciences Division, Senior Member, AIAA

Copyright (C) 1996 by the American Institute of Aeronautics and Astronautics, Inc. No copyright is asserted in the United States under Title 17, U.S. Code. The U. S. Government has a royalty-free license to exercise all rights under the copyright claimed herein for Government Purposes. All other rights are reserved by the copyright owner.
}

$\begin{array}{ll}\text { C.P. } & \text { Center of Pressure, m } \\ \text { D } & \text { drag, N } \\ \text { DAC } & \text { DSMC Analysis Code } \\ \text { DSMC } & \text { Direct Simulation Monte Carlo } \\ \text { L } & \text { vehicle reference length, } \mathrm{m} \\ \text { L/D } & \text { lift-to-drag ratio } \\ \mathrm{n} & \text { number density, molecules } / \mathrm{m}^{3} \\ \text { NURBS } & \text { Non-Uniform Rational B-Spline } \\ \mathrm{P} & \text { pressure, N/m }{ }^{2} \\ \text { q } & \text { heat transfer, W/m }{ }^{2} \\ \text { RLV } & \text { Reusable Launch Vehicle } \\ \text { SSTO } & \text { Single-Stage-To-Orbit } \\ \text { T } & \text { temperature, K } \\ \text { V } & \text { velocity, m } / \mathrm{s} \\ \text { VHS } & \text { Variable Hard Sphere } \\ \rho & \text { mass density, } \mathrm{kg} / \mathrm{m}^{3} \\ \tau & \text { shear stress, N/m }{ }^{2} \\ & \\ \text { Subscripts: } & \\ \infty & \text { freestream condition } \\ & \end{array}$

\section{Introduction}

The NASA/Air Force/industry Reusable Launch Vehicle (RLV) Technology Program ${ }^{1}$ has identified a number of technologies that are important to the goal of developing an all rocket-powered Single-Stage-To-Orbit (SSTO) vehicle. In the area of aerothermodynamics, analysis tools will be needed in both the continuum and the rarefied hypersonic regimes for predicting aerodynamics and heating. These analysis tools will be increasingly relied upon to provide data that historically have been obtained from ground-based and flight tests in order to reduce the overall development-cycle costs.

In the rarefied regime, direct simulation Monte Carlo (DSMC) codes will be required for predicting vehicle aerodynamics and for predicting reaction control 
system (RCS) plume interactions. In addition to the increasing computational demands that these analyses will place on DSMC, the DSMC codes should be increasingly flexible and easy to use. Since it may be necessary to couple the DSMC analyses with continuum CFD analyses, attention has recently focused on the appropriate grid and geometry methodologies for these tasks.

A variety of both body-fitted and Cartesian grid schemes have been used for DSMC simulations with Cartesian schemes receiving more attention in recent years. Several of these schemes are described in Ref. 2 for both two- and three-dimensional applications. Each scheme has advantages and disadvantages. Body-fitted grids can be aligned more naturally with the surface geometry which may facilitate the implementation of boundary conditions, and with structured body-fitted grids, the local coordinate directions may be altered to be better aligned with physical gradients in the flow than with Cartesian grids. However, it is possible to approximate many of the features of body-fitted grids through clustering of Cartesian elements. ${ }^{3-4}$ Body-fitted grids can in principle capture vehicle geometry more accurately than methods that approximate the surface as a set of discrete Cartesian elements. Unstructured body-fitted grids based on triangular elements in 2-D and tetrahedral elements in 3-D have a further advantage in that the grid resolution can be refined locally without altering the overall cell data structure. On the other hand, body-fitted grids are more computationally expensive for DSMC calculations because of the geometric computations required to track molecules crossing cell boundaries. Furthermore, the generation of the grid itself can be quite time consuming and can require significant effort on the part of the engineer to set up an appropriate grid for a particular problem.

Cartesian grids have been used for a variety of problems in computational physics and were first explored for three-dimensional DSMC simulations by McDonald ${ }^{5}$ primarily as a means of speeding up the calculations. Uniform Cartesian grids offer extremely efficient methods for locating a molecule within the grid by converting the molecule's position to an integer through a simple set of arithmetic operations. These indexing methods can be extended to nonuniform Cartesian grids without a major loss in efficiency by applying simple analytical stretching functions independently to each of the Cartesian coordinates. Furthermore, unstructured Cartesian grids can be constructed where the cells are formed by collections of Cartesian elements, ${ }^{3}$ and these too can be used in a relatively efficient manner through the use of run-length encoding or clever indexing schemes. Unstructured
Cartesian grids also provide an extremely flexible approach for grid adaption and ease of use in describing the geometry. ${ }^{4}$ At the same time, there are inherent difficulties in describing the geometry accurately in a Cartesian grid because the body lines do not conform to the grid. These difficulties become more significant for low-Knudsen-number, near-continuum flows where the required resolution near the body becomes extremely small relative to that required in the undisturbed stream. This may require modification to the basic grid scheme such as overlaying a local body-fitted mesh to resolve gradients normal to the wall. ${ }^{6}$

The purpose of this paper is to compare unstructured, body-fitted and Cartesian grid methodologies for application to low-density, hypersonic flows about a representative RLV geometry. A recent paper by Nance, et. al. ${ }^{7}$ compares the performance and accuracy of the unstructured, body-fitted code to that of a high-performance, parallel version of a uniform Cartesian grid code for relatively simple geometries. The present comparisons focus on the accuracy, efficiency, and ease of use of each approach for DSMC computations about a generic single-stage-to-orbit (SSTO) concept for which continuum flowfield solutions at lower altitude flight conditions have been presented. ${ }^{8}$ The body-fitted DSMC code has been adapted from an unstructured, tetrahedral grid code used by Celenligil and Moss. ${ }^{9}$ The Cartesian grid DSMC code is a new code under development by one of the authors (GJL) that uses a structured-grid, variable resolution approach. The modifications to and unique features of these codes are described, and the overall methodology for applying these codes to complex geometries is discussed. Comparisons of the DSMC results for the SSTO configuration obtained with these two codes are presented in terms of the aerodynamics and flowfields for a portion of a reentry trajectory in the neighborhood of $120 \mathrm{~km}$.

\section{Geometry}

A principal goal for the current body-fitted and Cartesian codes is that they have a common geometry specification with a great deal of geometric flexibility in order to handle complex geometries. However, it is also desirable that the codes not use a highly specialized data format that requires a labor intensive effort to convert from traditional CAD generated surfaces. To accomplish this goal, the surface geometry is specified as a collection of planar triangular elements which form an unstructured triangular grid. While it may provide more generality to specify the surface geometry using CAD-related definitions, such as NURBS (Non-Uniform Rational B-Spline), the unstructured triangular surface 
definition is much more computationally efficient. ${ }^{10}$ This is because of the difficulties associated with determining general 3-dimensional intersections of line segments, either flowfield-grid line intersections or molecule trajectories, with NURBS-defined surfaces.

The trade-off between geometric generality and computational efficiency is thought to be acceptable in that there are a number of grid generation packages which can easily create unstructured triangular surfaces from CAD-defined NURBS patches. An additional benefit of the unstructured surface grid definition is that the surface sampling elements for momentum and energy exchange are naturally defined by each triangle, and this provides some control over the surface sampling resolution that is independent of the volume grid. Each of the triangles can additionally have a uniquely specified surface temperature or outgassing flux. This allows a completely general method of defining uniform or non-uniform surface boundary conditions or molecule inflow properties on 3-dimensional surfaces such as on continuum breakdown surfaces for rocket engine plumes. $^{11}$

In the body-fitted, tetrahedral code, the surface triangles form one face of the tetrahedra adjacent to the surface, and the volume grid is constructed using advancing front techniques. ${ }^{12}$ For the Cartesian code, it is desired to minimize the computational effort required to determine molecule-surface interactions, so the surface triangles are mapped to the Cartesian cells. Then only those molecules which reside in cells that are cut by the triangulated surface need be tested for an interaction. The reduced volume of each cell clipped by one or more triangles is computed, and provision is made for cells to be fragmented into two or more subvolumes which might occur with thin surfaces.

The geometry for the SSTO configuration used in this study is the same as that used in Ref. 8 with zero deflection control surfaces and is shown in Fig. 1. This geometry was received as a 161 x 129 structured surface grid (Fig. 2) which was triangulated using the FELISA ${ }^{12}$ unstructured grid generation software to produce an unstructured surface grid such as that shown in Fig. 3. This triangular, unstructured surface grid was used as the surface geometry description for both the body-fitted and Cartesian grid calculations. For the unstructured, body-fitted grid code, an unstructured, tetrahedral volume grid was also generated using the FELISA software where the triangular, unstructured surface grid forms the faces of the tetrahedra adjacent to the surface. For the Cartesian grid calculations, the triangulated surface grid was used only to represent a collection of triangles that cut through the Cartesian mesh. However, since both codes used the same unstructured surface grid, the surface sampling elements are identical.

\section{DSMC Method}

The DSMC method is well documented in the literature and a recent book ${ }^{2}$ describes the method as well as the various grid methodologies that have been used. The DSMC method is based on tracking the movement of thousands to millions of representative molecules as they move through physical space while undergoing collisions among themselves as well as with any surface boundaries that may be present. A grid is superimposed on the computational region of interest and in principle is needed only to facilitate the selection of nearest-neighbor collision pairs and to provide cells within which samples can be taken for computing macroscopic properties such as density, velocity and temperature.

Collisions in the present DSMC codes are modeled using the Variable Hard Sphere (VHS) model. ${ }^{2}$ For molecules having internal degrees of freedom, the Larsen-Borgnakke ${ }^{13}$ phenomenological model is used to repartition energy among the internal and translational modes after a collision. Gas-surface interactions are modeled using fully diffuse scattering with full thermal accommodation.

The role of the grid in tracking the motion of the molecules depends on the type of grid. For grids in which the cell faces are polygons that are allowed to have an arbitrary orientation with respect to the coordinate axes, the molecular motion generally must be tracked using ray-tracing-like procedures. The geometrical calculations required to determine whether a molecule leaves a particular cell are floating-point intensive, and in general, it is possible that multiple molecular-trajectory/cell-face intersection calculations may be required during a single time step. One of the principal advantages of the Cartesian grids is that many of these ray-tracing calculations can be eliminated, because once the final position of the molecule is known, the index of the cell to which the molecule belongs can be easily calculated. However, as the Cartesian codes are made more general by allowing the time step to vary over the domain, some of these advantages disappear. It is also necessary with Cartesian grids to check for possible collisions with boundaries. Therefore, it is still necessary to use either ray-tracing where appropriate or some special knowledge of the geometry to check for surface collisions.

\section{Body-Fitted Grid Code}

The 3D body-fitted code used in the present study has been adapted from an existing unstructured, 
tetrahedral grid code. ${ }^{9}$ The code has many of the same features as the general two-dimensional DSMC code known as G2 developed by Bird ${ }^{14}$ and is referred to as the $\mathrm{G} 3$ code. The G3 code handles multiple species with chemical reactions and models collisions with the VHS and Larsen-Borgnakke models. The original code allowed the ratio of real-to-simulated molecules and the time step to vary from cell to cell but required that the ratio of the two quantities be the same for all cells. The code was modified to allow the ratio of real-to-simulated molecules and the time step to vary independently, but this feature was not used for the current calculations, and the ratio of these two quantities was held constant from cell to cell. This version also treats each tetrahedron as an individual cell; however a version exists that allows the tetrahedra to be treated as subcells within an overall structured, body-fitted grid. ${ }^{9}$ The code provides for an arbitrary unstructured tetrahedral grid to be used as input.

The $3 \mathrm{D}$ code was modified to provide a number of enhancements in both performance and ease of use. Preprocessing software was written to allow the direct use of the unstructured, surface and volume grid files generated by the FELISA software. The ray-tracing procedures were then modified to be more efficient by precomputing many of the cell-face geometrical quantities needed to perform the logical tests that determine when a molecule crosses a cell face. A number of other minor improvements were made to reduce the total number of floating-point computations in the code and to improve the accuracy in tracking molecules through the grid. Postprocessing software was written to allow both the surface and flowfield properties to be examined using standard plotting software. A simple, 3D free-molecular code was also written that accepts the same FELISA-generated, unstructured surface mesh for computing surface properties and overall aerodynamic quantities. Finally, all preprocessing, run-time, and postprocessing software were rewritten to use dynamic memory allocation, so that all of the steps required to generate the grid, run the simulation, and generate final results for plotting may be run without requiring any recompilation of source code. One of the difficulties in previous applications of the original code was the excessive time required to generate the grid and preprocess it into the form needed by the DSMC code. With the present codes, these difficulties have been significantly reduced. With the present suite of executable codes, it is possible to start from a given surface geometry (described as a surface mesh) to the start of the DSMC simulation in a matter of minutes on a typical engineering workstation.

A typical unstructured grid for the body-fitted calculations is shown in Fig. 4. Only the mesh on the outer flow boundary, symmetry plane and vacuum outflow boundary are shown for clarity. Since calculations are presented only for zero sideslip angle, a symmetry boundary condition is imposed on the symmetry plane of the vehicle and only one-half of the vehicle geometry is required for the simulations. The outer flow boundary is treated as a uniform stream boundary, and the outflow boundary is treated as a vacuum. The outflow boundary is located at the axial location corresponding to the trailing edge of the wings and the fuselage base.

\section{Cartesian Grid Code}

The present Cartesian grid DSMC code is an attempt to merge what have proven to be successful strategies for previous DSMC codes with new techniques and to integrate them into a new algorithm named DAC (DSMC Analysis Code). The design goal for DAC is to develop efficient procedures for the simulation of general rarefied gas dynamics problems, and to that end the following strategies have been adopted and tested.

The computational domain of the flowfield is bounded by a rectangular Cartesian box with user specified limits. The computational domain is divided into a "typical" Cartesian network of cells with constant, yet independent, spacing in each of the three coordinate directions. The cells of this first level of Cartesian refinement are referred to as level-1 cells. The resolution of this grid is typically set based on the minimum desired flowfield sampling resolution for a given problem. To further refine the flowfield grid in areas of increased density or high gradients, each level-1 cell can have an additional level of embedded Cartesian refinement. This second level of refinement is independent for each level-1 cell and is also variable in each of the three coordinate directions should refinement in a specific direction be desired. A typical Cartesian mesh for an SSTO simulation with $1 \times 1 \times 1$ resolution in the outer regions that has been refined based on adapting to a solution obtained using uniform $1 \mathrm{x} 1 \mathrm{x} 1$ resolution in every level-1 cell is shown in Fig. 5. After adaption, the resolution near the windward surface of the vehicle is 10 times greater than that used in the outer level-1 cells.

The ability to refine the flowfield grid locally allows DAC to meet the spatial resolution requirement of the DSMC method without excessive global refinement. An additional requirement of DSMC is that there should be sufficient molecules in each cell for obtaining accurate collision statistics. ${ }^{2}$ However, for computational efficiency, one would also like to have the same number of molecules in each cell which is difficult to achieve simply through local cell refinement 
when there is a highly nonuniform density distribution in the computational domain. At least a partial solution to this latter problem can be obtained by using variable scaling of real-to-simulated molecules throughout the domain in a manner similar to that used by Bird for the G2 code $^{14}$ In the current version of DAC, the ratio of real-to-simulated molecules is allowed to vary from one level- 1 cell to the next. The time step is also allowed to vary for the level-1 cells independently of the real-to-simulated molecule ratio so that an appropriate local time step may be applied in all level-1 computational cells. A consequence of varying the ratio of real-to-simulated molecules and the time step independently is that a balance of flux is no longer guaranteed when a molecule moves from one level-1 cell to the next. This requires that steps be taken to adjust the flux accordingly to maintain the balance. This is accomplished by appropriately cloning or deleting molecules as the simulation molecules cross level- 1 cell boundaries. To minimize undesirable statistical effects on the collision statistics caused by molecule cloning, a delay buffer is implemented so that, on average, cloned molecules are introduced to the flowfield with a random delay such that the probability of having a cloned pair in the same cell during a given time step is less than some user specified value.

The determination of appropriate flowfield grid resolution, mapping the surface geometry to the flowfield grid and scaling the time steps and ratios of real-to-simulated molecules are currently handled by a preprocessing code. In the input file for the preprocessor, the user simply specifies the freestream conditions, computational domain parameters, and gas and surface properties. The preprocessor then automatically determines the appropriate level of secondary refinement based either on the number density of the specified freestream or optionally based on the local number density from a previous solution.

The entire process of obtaining a solution for a given problem is designed to require very little effort on the part of the user once the triangulated surface definition is obtained. This allows minimal expertise in the DSMC code methodology to be required of the user. The input file for DAC itself requires little more than setting the number of time steps the code should run, the flowfield sampling interval and the interval for writing intermediate data files. Postprocessing software allows the user to generate files for viewing surface or flowfield properties using standard plotting software. Dynamic memory allocation is used for all variable arrays whose size is dependent on the runtime parameters.

The present version of DAC only models the rotational mode for internal energy of polyatomic species. For the high-altitude problem to be considered, the vibrational mode is not sufficiently excited to contribute significantly in determining either the flowfield or surface properties. This was confirmed by examining the degree of vibrational excitation computed with the more general body-fitted grid code. However, it is expected that vibrational relaxation and chemical reaction modeling will be included in a future version of DAC.

\section{Computational Conditions}

DSMC simulations have been performed for an altitude of $120 \mathrm{~km}$ based on the reentry trajectory given in Ref. 8. The nominal freestream conditions are:

$$
\begin{aligned}
& \text { Velocity }=7817.9 \mathrm{~m} / \mathrm{s} \\
& \begin{aligned}
& \text { Number density }=5.107 \times 10^{17} \\
& \text { molecules } / \mathrm{m} 3
\end{aligned}
\end{aligned}
$$

Temperature $=360.0 \mathrm{~K}$

and the vehicle is at an attitude of 32.0 degrees angle of attack. Simulations were also performed for angles of attack of 10, 20, 40, and 50 degrees with the tetrahedral code to provide additional low-density aerodynamic data on the SSTO configuration.

The freestream air consists of $0.0863,0.7316$, and 0.1821 mole fractions of $\mathrm{O}_{2}, \mathrm{~N}_{2}$, and $\mathrm{O}$ respectively. The VHS parameters are based on viscosity-temperature curve fits for $\mathrm{N}_{2}$ at a reference temperature of $2880 \mathrm{~K}$. Preliminary calculations were also performed using Argon as the freestream gas, but the flowfield and surface results are qualitatively similar to those for air at this rarefied condition, and therefore, only the air results are included in this paper. Similarly, simulations which included chemistry showed little effect at the present conditions, and all results are shown only for nonreacting air. The surface temperature is assumed to be $1000 \mathrm{~K}$. Based on a post-calculation radiative equilibrium analysis, this temperature is unrealistically high for much of the body, but it is chosen to be representative of the surface temperature near the stagnation point and is assumed to be uniform over the body. All calculations assume fully diffuse reflection with complete accommodation.

Based on the given freestream conditions and the nominal body length of $56.57 \mathrm{~m}$ for the SSTO configuration, the overall Knudsen number is about 0.08 .

\section{$\underline{\text { Results }}$}

A typical flowfield obtained with the Cartesian grid code is illustrated in Fig. 6 by translational temperature contours. While the translational temperatures appear to 
be very high near the stagnation point on the nose of the vehicle, these temperatures are mainly representative of the bimodal velocity distribution resulting from superimposing freestream and reflected molecules rather than representative of the collisionality of the flow. Similar results were obtained with the body-fitted grid code, and a comparison of the tetrahedral and Cartesian code results for number density and translational and internal temperatures along an approximate stagnation streamline is shown in Fig. 7.

Comparisons of surface contours of pressure, shear stress and heat transfer obtained with the two codes are shown in Fig. 8 for both the upper (leeward) and lower (windward) surfaces of the body. The results match extremely well both qualitatively and quantitatively for all three quantities. While there is more scatter in the data on the upper leeward side, the two codes still agree very well. This scatter is partly due to the low resolution (large surface elements) used on the leeside as well as to the extremely low densities that occur in the wake. For the freestream conditions used, the actual surface fluxes are up to 10,000 times smaller on the leeside than near the stagnation point. Therefore, even though the flowfield cells in the wake may have a reasonable number of simulated molecules, the relative number of simulated molecules hitting the surface is still much less on the leeside than on the windward side.

Quantitative comparisons of the distributions of pressure, shear stress, and heat transfer along the windward and leeward centerlines are shown in Figs. 9-10. The leeside quantities generally fall to extremely low values such that the leeside forces and heat transfer do not contribute significantly to the overall aerodynamic forces and heat transfer for the selected flight condition. Fig. 9 is a comparison of results obtained with the body-fitted code with collisions inhibited (collisionless DSMC) to those obtained with a free-molecular analysis. This comparison is shown simply to demonstrate that the basic algorithm used for tracking molecular motion gives the correct results in the free-molecular limit. Fig. 10 shows a comparison of the tetrahedral and Cartesian codes with collisions enabled. Excellent agreement between the two codes is obtained. By comparing Figs. 9 and 10, it is seen that while the pressure is relatively unchanged due to flowfield collisions, the shear stress (friction) and heat transfer are significantly lower than those predicted for free molecular flow.

A comparison of typical aerodynamic quantities obtained from DSMC, collisionless DSMC, free molecular, and modified Newtonian calculations is shown in Fig. 11. Fig. 11(a) gives the lift-to-drag (L/D) ratio, Fig. 11(b) gives the center-of-pressure (C.P.) location nondimensionlized by the nominal body length, and Fig. 11(c) gives the pitching moment coefficient relative to the center-of-gravity (C.G.) of the vehicle. The results shown were obtained with the tetrahedral code. However, similar quantities obtained with the Cartesian code at an angle of attack of 32 degrees agreed to within 1-2 percent.

Although it is not evident because the scale of Fig. 11(a) is distorted by the large L/D predicted for Newtonian flow, the collisionless DSMC and free molecular values at an angle-of-attack of 32 degrees agree to within 0.1 percent. The DSMC result (with collisions enabled) is higher than the free molecular value but is significantly lower than the Newtonian limit at this Knudsen number. Although one might expect the L/D to be even higher at this Knudsen number, it should be noted that it is usually more appropriate to base the Knudsen number on the mean aerodynamic chord length for a wing-body geometry such as the SSTO configuration in which case the "effective" Knudsen number is probably closer to unity which is indicative of a more rarefied flow. The higher L/D predicted with DSMC is mostly due to the reduced surface friction relative to the free-molecular result.

Figs. 11(b) and (c) show that the center-of-pressure is ahead of the C.G. for this flight condition resulting in a positive (nose-up) pitching moment at all angles of attack for both the free-molecule and DSMC results. This is in contrast to the nose-down pitching moment predicted for the Space Shuttle with similar control deflections and under similar flight conditions. ${ }^{6}$ Also, the DSMC results differ only slightly from the free-molecule results further indicating the overall rarefied nature of the flow. However, it should be noted that the absolute magnitude of the pitching moment predicted by both DSMC and free-molecular analyses is very small, and only a small force would be required to trim the vehicle at any of the attitudes shown.

\section{$\underline{\text { Discussion }}$}

From the results shown, it is obvious that comparable results can be obtained with both grid methodologies. In fact, both the tetrahedral and Cartesian codes have been used to augment the predictions of Rault ${ }^{6}$ and all three code results compare favorably with aerodynamic flight data for the Shuttle Orbiter. $^{15}$

However, the principal differences in the two methodologies lie not in their accuracy but more in their relative ease of implementation and application. For example, the tetrahedral code requires the initial generation of a volume grid whereas the Cartesian code requires only the surface triangulation. Furthermore, the 
tetrahedral code currently does not have the ability to perform grid adaption based on a previous solution, whereas the Cartesian code can refine each of its Cartesian level-1 cells using a single independent level of refinement in each direction. In principle, it is straightforward to perform local refinement of the tetrahedral grid, and adaptive refinements of triangular meshes have been used successfully in 2-D. ${ }^{16}$ However, because each tetrahedron may have an arbitrary orientation with respect to the local flow gradients, the "effective" dimension of the cell depends on the orientation. Furthermore, it has been observed that topological difficulties in the initial unstructured grid generation can often produce tetrahedra with undesirably large aspect ratios.

Notwithstanding the difficulties with grid adaption, the unstructured, tetrahedral grid would appear to have certain advantages in that the basic cell data structure remains the same when new nodes and new cells are added to the node and connectivity lists respectively. Similar advantages could be achieved with an unstructured Cartesian grid where the cell has a "brick" geometry and a cell data structure similar to the tetrahedral grid. However, then many of the advantages such as fast indexing offered by more structured Cartesian grids would be lost. With the present Cartesian code, it is possible to add additional levels of refinement, but such modifications require some additional complexity to the indexing schemes used to track molecule movement. ${ }^{2}$ Unstructured, tetrahedral grids may also have certain topological advantages for efficient allocation of cells for internal flows involving very complex geometries.

It is also clear that the Cartesian schemes are inherently faster on a per molecule per time step basis. Because the actual performance differences depend strongly on the nature of the problem, the grid, and the user-defined computational time step, it is difficult to make an accurate assessment of the differences. Based on limited timings for some of the present calculations and those of Nance, ${ }^{7}$ it appears that the computational time per molecule per time step is typically 2-10 times less with the current Cartesian method than with the tetrahedral method. However, with optimal grid refinement and local number density scaling, it is possible in many cases to perform accurate simulations of entry vehicle aerodynamics using comparable CPU and memory resources with both methods.

Finally, although the Cartesian schemes appear to offer the most advantages, it seems clear that no single grid methodology is universally superior for all problems. In fact, it is likely that a hybrid scheme in which a Cartesian grid is used for the bulk of the problem together with a local body-fitted grid near the body may offer significant advantages for near continuum solutions about RLV type vehicles.

\section{Summary}

Two different grid methodologies for DSMC simulations have been applied to the prediction of a hypersonic, rarefied flow about a representative reusable launch vehicle (RLV) geometry. An unstructured, tetrahedral grid code and a structured, variable-resolution Cartesian grid code give results that agree extremely well for both surface and flowfield quantities as well as for overall aerodynamic and heating behavior.

Both codes use "off-the-shelf" software for geometry and grid generation. The Cartesian code typically requires less initial work to set up a DSMC simulation and is more computationally efficient than the tetrahedral code. However, the unstructured, tetrahedral approach offers certain advantages for grid refinement, although this is due simply to the unstructured data structure rather than the grid geometry. While the Cartesian method appears to offer greater overall advantages, both methods are suitable for predicting entry aerodynamics of RLV-type configurations in the rarefied, transitional regime.

For the $120 \mathrm{~km}$ conditions simulated, the flowfield is very rarefied, but surface properties on the single-stage-to-orbit RLV configuration show significant departure from those predicted for free-molecular flow. In particular, the shear stress and heat transfer are significantly lower on the windward side of the vehicle than the corresponding free-molecular values. The lower shear stress results in a higher lift-to-drag ratio and a slightly higher pitching moment than predicted with the free-molecular model, but much lower lift-to-drag ratio and a totally different pitching moment behavior than predicted based on modified Newtonian theory.

\section{References}

1. Access to Space Study -- Summary Report, Office of Space Systems Development, NASA Headquarters, January 1994.

2. Bird, G. A.: Molecular Gas Dynamics and the Direct Simulation of Gas Flows, Clarendon Press, Oxford, 1994.

3. Bird, G. A.: "Application of the Direct Simulation Monte Carlo Method to the Full Shuttle Geometry," AIAA Paper 90-1692, 1990.

4. Rault, D. F. G.: "Towards an Efficient Three Dimensional DSMC Code for Complex Geometry Problems," 18th Rarefied Gas Dynamics Symposium, July 1992. 
5. McDonald, J. D., and Baganoff, D.:

"Vectorization of a Particle Simulation Method for Hypersonic Rarefied Flows," AIAA Paper 88-2735, June 1988.

6. Rault, D. F. G., "Aerodynamics of the Shuttle Orbiter at High Altitudes," J. of Spacecraft and Rockets, vol. 31, 1994, pp. 944-952.

7. Nance, R. P., Wilmoth, R. G., and Hassan, H. A.: "A Comparison of Grid-Defintion Schemes for DSMC," AIAA Paper 96-0604, 1996.

8. Weilmuenster, K. J., Gnoffo, P. A., Greene, F. A., Riley, C. J., and Hamilton, H. H., II: "Hypersonic Aerodynamic Characteristics of a Proposed Single-Stage-to-Orbit Vehicle," AIAA Paper 95-1850, June, 1995.

9. Celenligil, M. C. and Moss, J. N.: "Hypersonic Rarefied Flow About a Delta Wind - Direct Simulation and Comparison with Experiment," AIAA Journal, vol. 30, 1992, pp. 2017-2023.

10. Melton, J. E., Berger, M. J., Aftosmis, M. J., and Wong, M. D.: "Development and Application of a 3D Cartesian Grid Euler Method," in Surface Modeling, Grid Generation, and Related Issues in Computational Fluid Dynamic (CFD) Solutions, NASA CP-3291, 1995, pp. 225-250.

11. Lumpkin, F. E., III, LeBeau, G. J., and Stuart, P. C.: "A CFD/DSMC Analysis of Plumes and Plume Impingement During Shuttle/Mir Docking," AIAA Paper 95-2034, June, 1995.

12. Peraire, J., Morgan, K., and Peiro, J.:

"Unstructured Finite Element Mesh Generation and Adaptive Procedures for CFD," in Application of Mesh Generation to Complex 3-D Geometries, AGARD CP-464, 1990, pp. 18.1-18.12.

13. Borgnakke, C., and Larsen, P. S.: "Statistical Collision Model for Monte Carlo Simulations of Gas Mixture," J. Computational Phys., vol. 18, 1975, pp. 405-420.

14. Bird, G. A.: "The G2/A3 Program System Users Manual," G.A.B. Consulting Pty Ltd, Killara, N.S.W., Australia, Mar. 1992.

15. Blanchard, R. C., Wilmoth, R. G., and LeBeau, G. J.: "Orbiter Aerodynamic Acceleration Flight Measurements in the Rarefied-Flow Transition Regime," AIAA Paper 96-2467.

16. Boyd, I., Kannenberg, K. C., and Dietrich, S.: "Parallel DSMC Computation of Planetary Probe Flow Using An Adapted Unstructured Grid," in Aerothermodynamics for Space Vehicles and High-Velocity Flow Data Base, edited by W. Berry, J.A. Desideri, F. Grasso, J. Muylaert, and J. Periaux, Wiley, 1996 (in press).

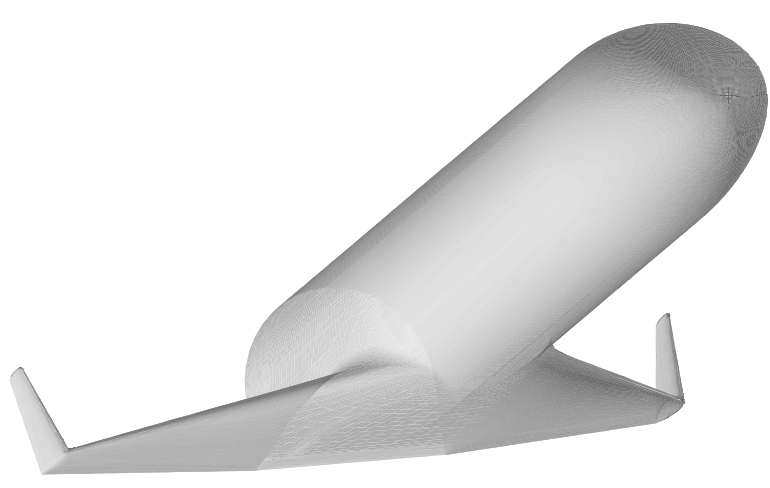

Fig. 1 - Single-stage-to-orbit surface geometry.

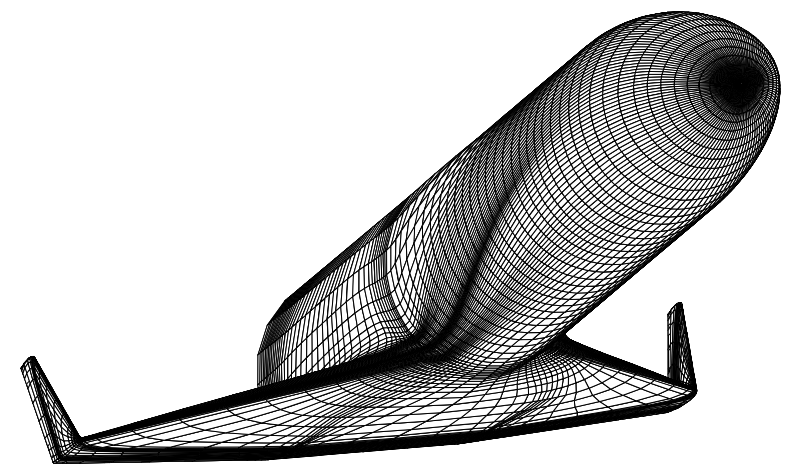

Fig. 2 - Surface grid used to define geometry for triangulation. 


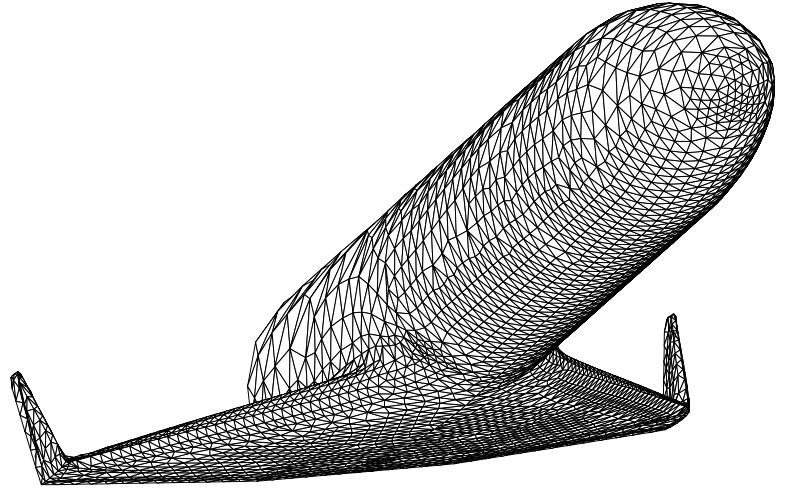

Fig. 3 - Typical triangulated surface mesh used with tetrahedral and Cartesian codes.

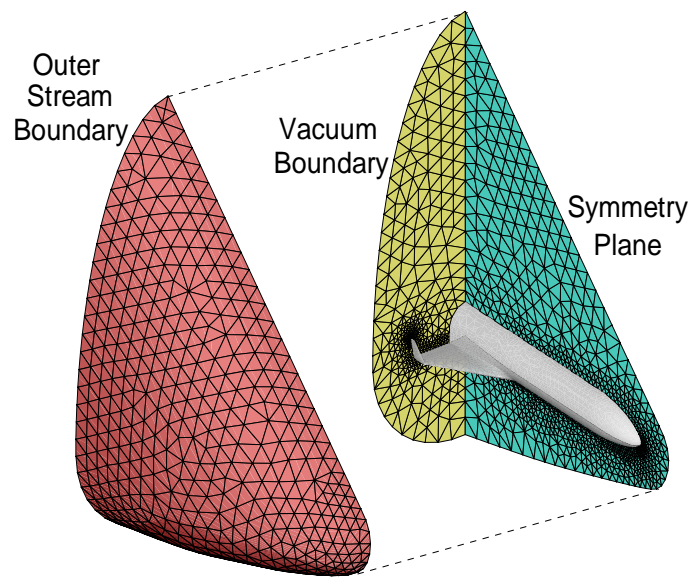

Fig. 4 - Typical boundary mesh for tetrahedral calculations.

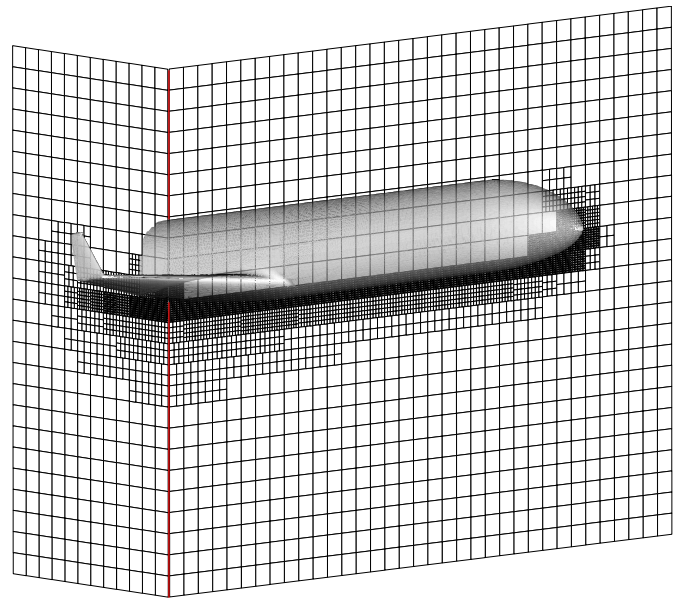

Fig. 5 - Typical mesh used by Cartesian code after flowfield adaption.

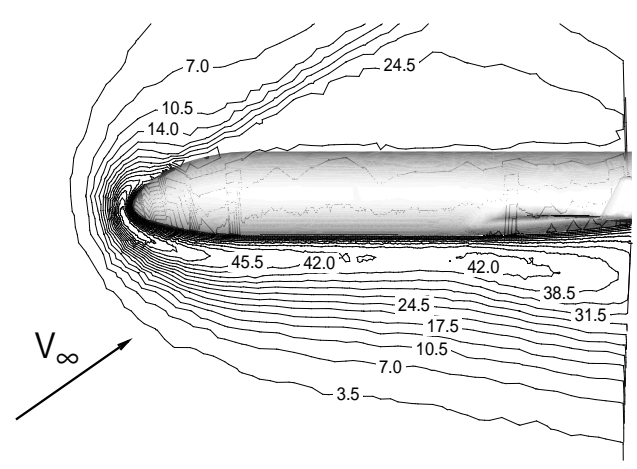

Fig. 6 - Translational temperature contours (nondimensionalized by freestream temperature). Computed with Cartesian code at $120 \mathrm{~km}$.

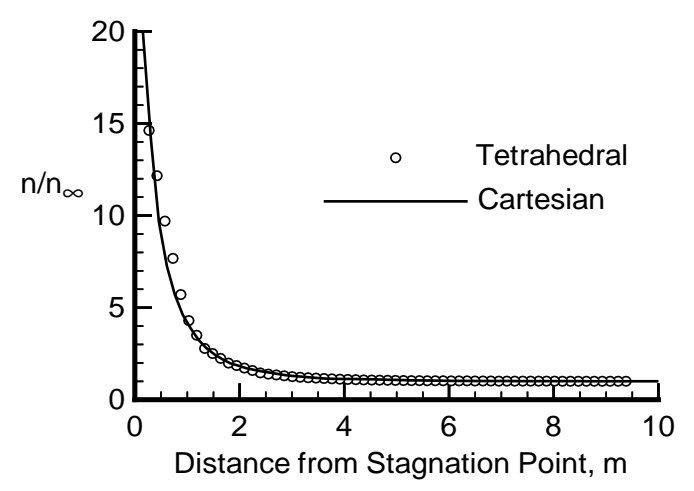

(a) Number density.

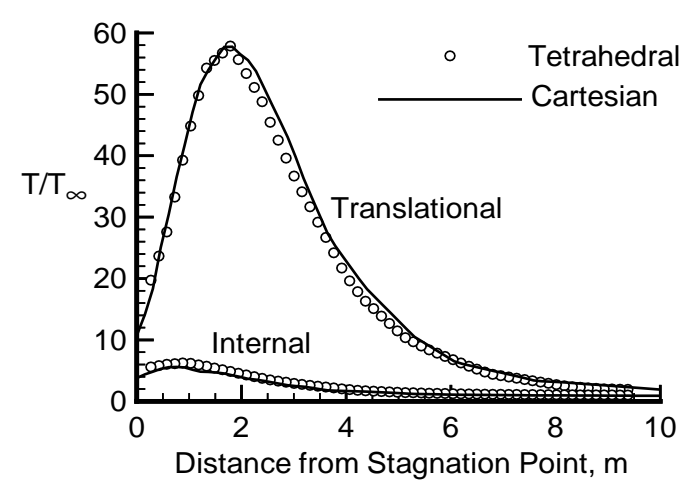

(b) Temperature.

Fig. 7 - Comparison of tetrahedral and Cartesian flowfield properties along approximate stagnation streamline. 

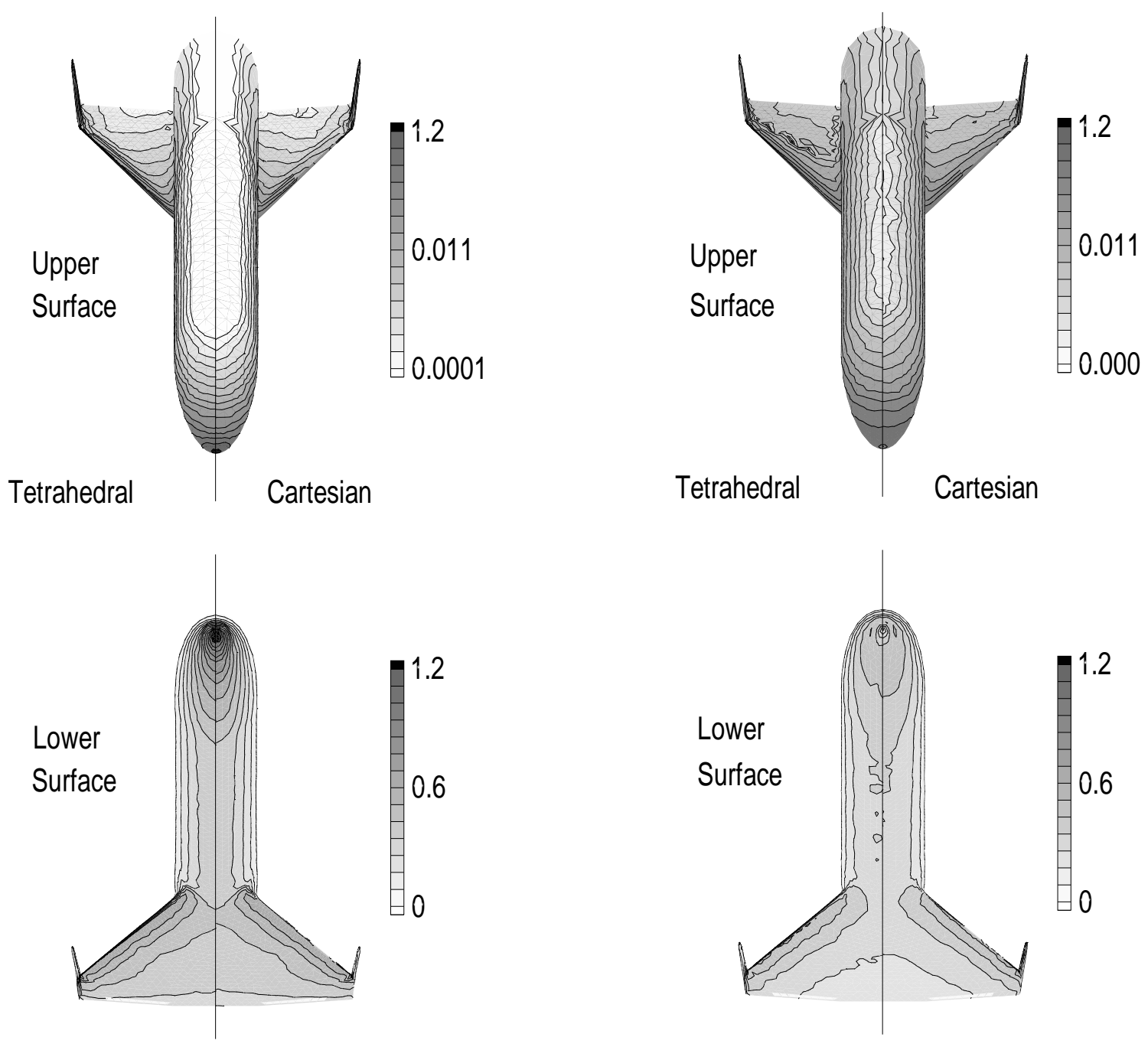

(a) Pressure.

(b) Shear stress.

Fig. 8 - Contours of properties on upper and lower surfaces.

Fig. 8 - Continued. 

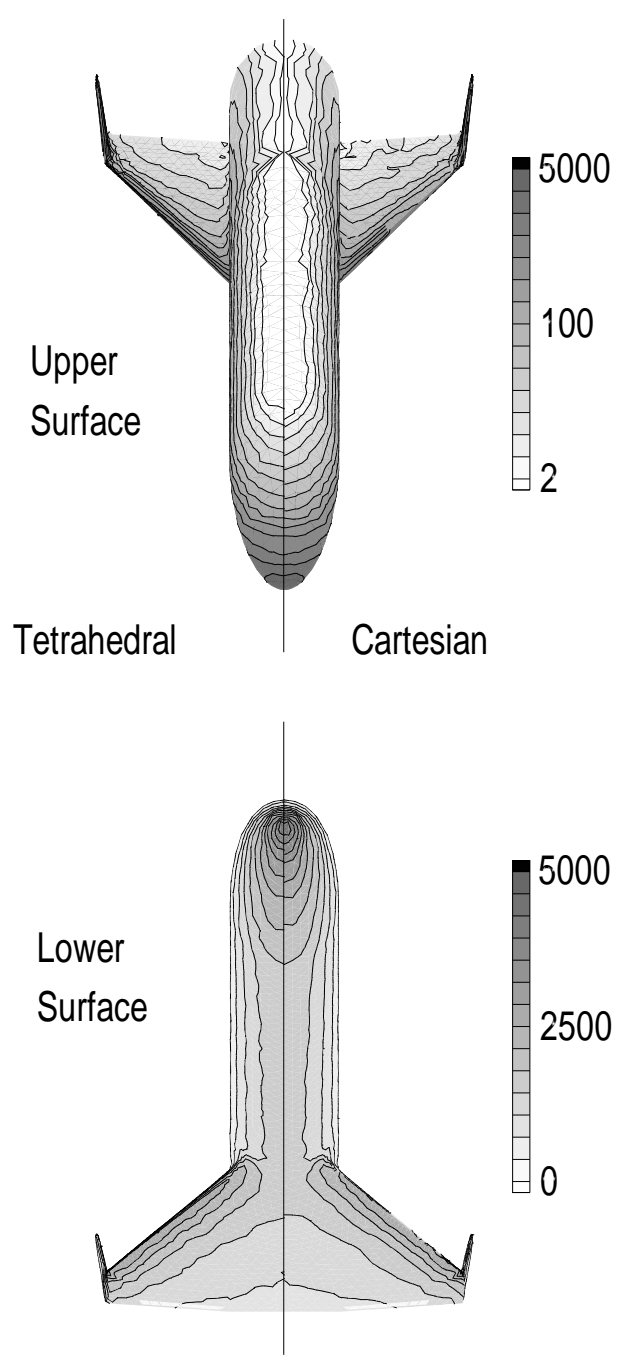

(c) Heat transfer.

Fig. 8 - Concluded.
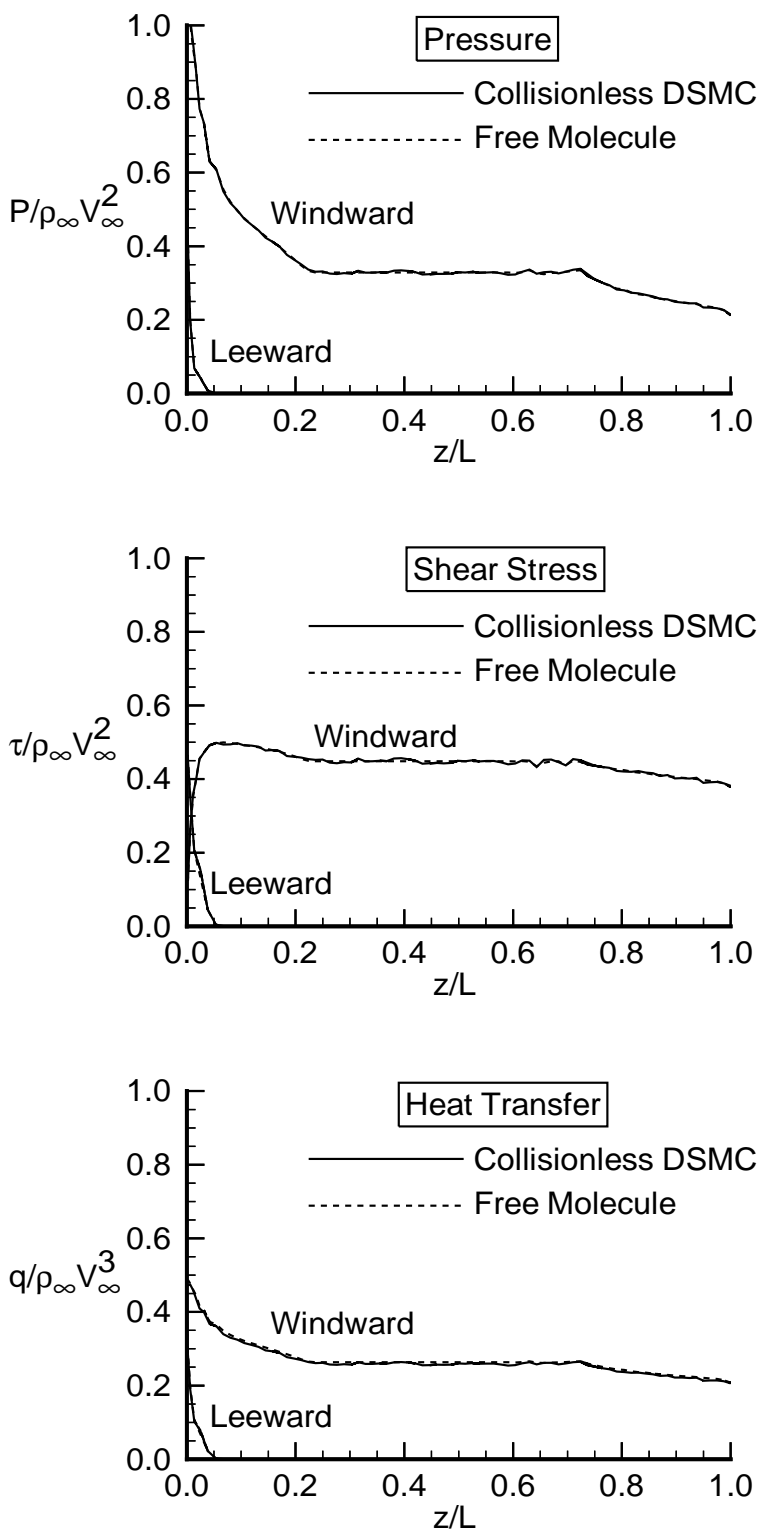

Fig. 9 - Comparison of surface properties near centerline for collisionless DSMC and free molecular calculations. 

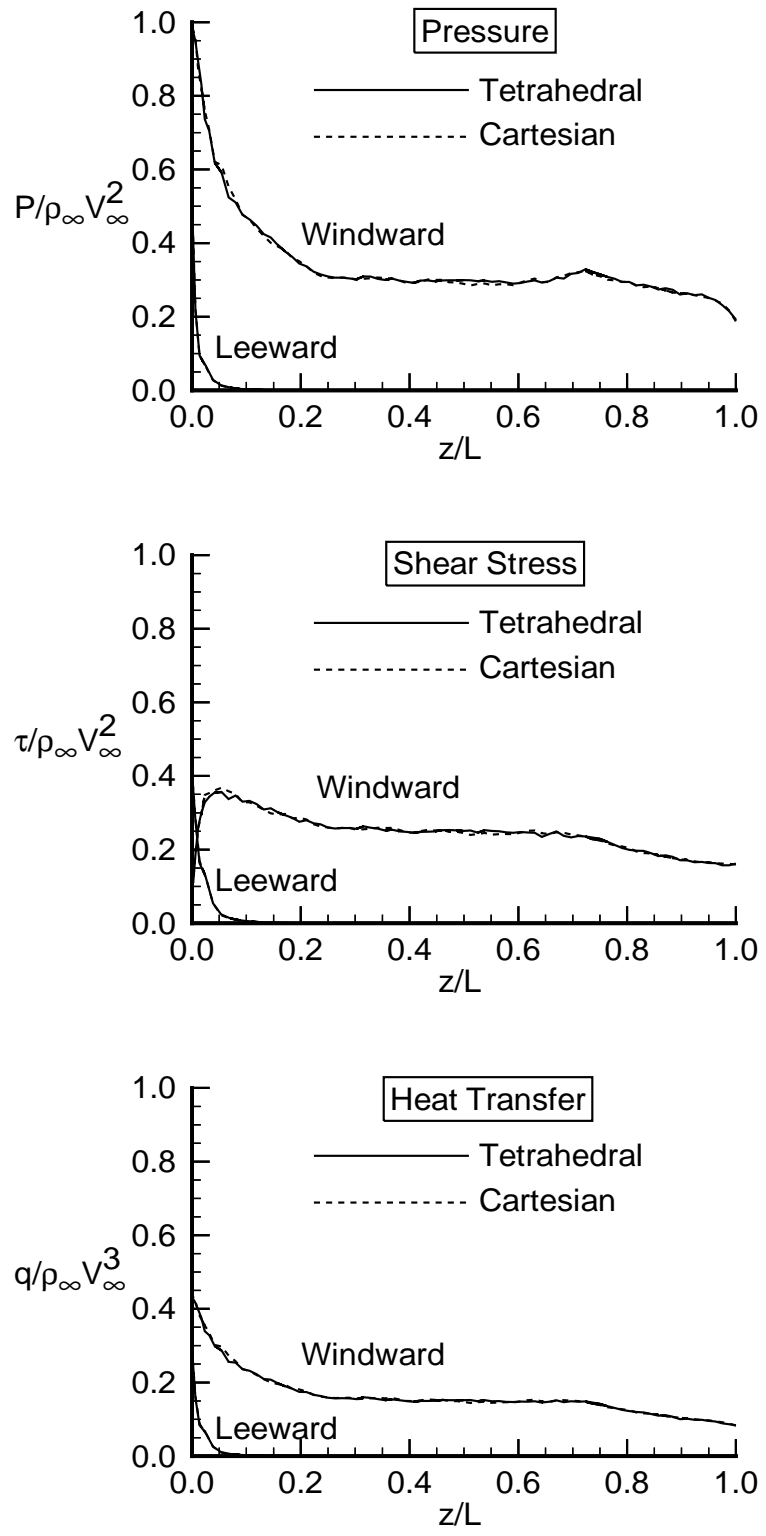

Fig. 10 - Comparison of surface properties near centerline for tetrahedral and Cartesian DSMC calculations.

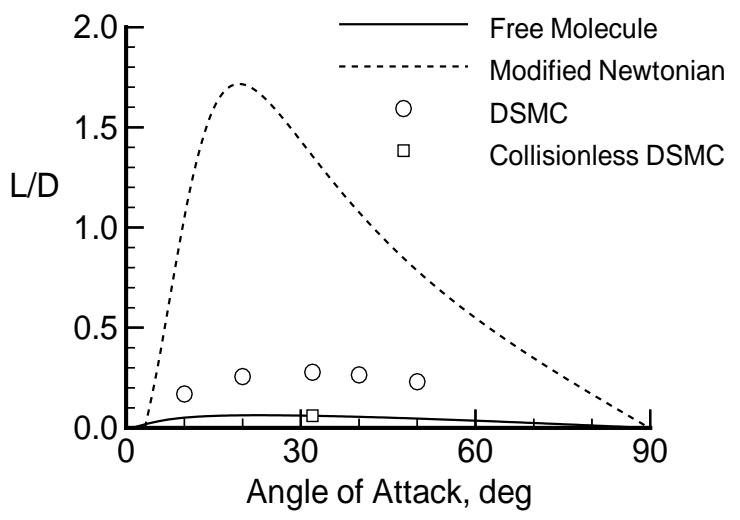

(a) Lift-to-drag (L/D) ratio.

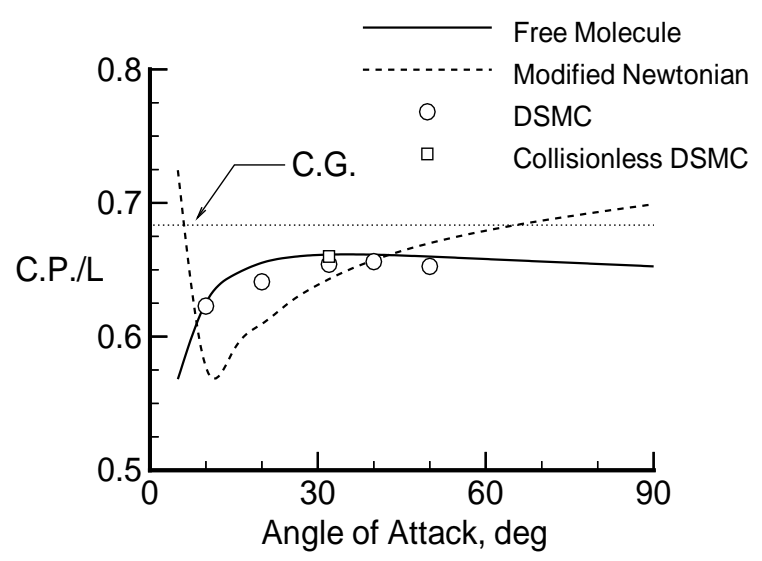

(b) Center of pressure.

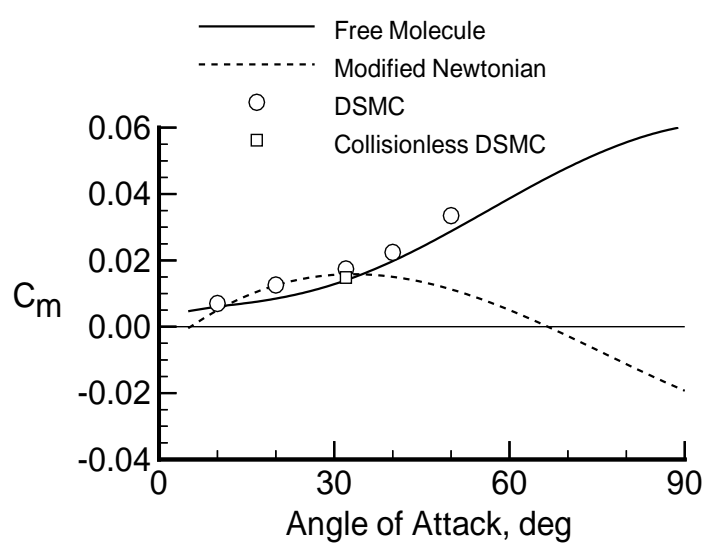

(c) Pitching moment about C.G.

Fig. 11 - Typical aerodynamic quantities computed with tetrahedral code. 